\title{
Exercising your fat (metabolism) into shape: a muscle-centred view
}

\author{
Anne Gemmink $^{1}$ - Patrick Schrauwen ${ }^{1}$ - Matthijs K. C. Hesselink ${ }^{1}$
}

Received: 3 February 2020 / Accepted: 23 March 2020 / Published online: 12 June 2020

(C) The Author(s) 2020

\begin{abstract}
Fatty acids are an important energy source during exercise. Training status and substrate availability are determinants of the relative and absolute contribution of fatty acids and glucose to total energy expenditure. Endurance-trained athletes have a high oxidative capacity, while, in insulin-resistant individuals, fat oxidation is compromised. Fatty acids that are oxidised during exercise originate from the circulation (white adipose tissue lipolysis), as well as from lipolysis of intramyocellular lipid droplets. Moreover, hepatic fat may contribute to fat oxidation during exercise. Nowadays, it is clear that myocellular lipid droplets are dynamic organelles and that number, size, subcellular distribution, lipid droplet coat proteins and mitochondrial tethering of lipid droplets are determinants of fat oxidation during exercise. This review summarises recent insights into exercise-mediated changes in lipid metabolism and insulin sensitivity in relation to lipid droplet characteristics in human liver and muscle.
\end{abstract}

Keywords Athletes · Exercise · Fat metabolism · Intramyocellular lipid droplets · Lipid droplet-mitochondria interaction · Lipid-droplet turnover $\cdot$ Liver $\cdot$ Muscle $\cdot$ Review $\cdot$ Type 2 diabetes

\author{
Abbreviations \\ ATGL Adipose triglyceride lipase \\ IHL Intrahepatic lipid \\ IMCL Intramyocellular lipid \\ PLIN Perilipin \\ $W_{\max } \quad$ Maximal power output
}

\section{Introduction}

During physical exercise, the increase in energy demand is fuelled by oxidation of glucose and fatty acids [1]. The relative and absolute contribution of glucose or fat oxidation is dependent on the prandial state (and substrate availability), exercise intensity and training status [2]. Endurance-trained athletes are

Electronic supplementary material The online version of this article (https://doi.org/10.1007/s00125-020-05170-z) contains a slideset of the figures for download, which is available to authorised users.

Matthijs K. C. Hesselink

Matthijs.hesselink@maastrichtuniversity.nl

1 Department of Nutrition and Movement Sciences, NUTRIM School for Nutrition and Translational Research in Metabolism, Maastricht University Medical Centre+, 6200 MD Maastricht, the Netherlands at the high end of the spectrum of fat oxidative capacity, whereas insulin-resistant individuals typically possess compromised fat oxidative capacity. In both populations, endurance training improved fat oxidative capacity. Fatty acids used during exercise can originate from the circulation, packed in triacylglycerol-rich particles originating from the liver or as NEFAs, predominantly originating from adipose tissue lipolysis [1]. The relative contribution of the different fat pools to whole-body fat oxidation is exercise-intensity dependent, with fat oxidation rising between $40 \%$ and $55 \%$ of maximal power output $\left(W_{\max }\right)$ and then declining towards $75 \% W_{\max }$. Tracer studies revealed that this comprises a drop in oxidation of NEFAs and triacylglycerol fat sources (intramyocellular lipid [IMCL] and lipoprotein-derived triacylglycerols) [3].

Other sources of exercise-fuelling fatty acids are the IMCLs, of which the majority is stored in triacylglycerolrich lipid droplets dispersed throughout the muscle [1]. The observation that in non-athletes, insulin sensitivity correlates negatively with IMCL content led to the suggestion that IMCL content directly impedes insulin sensitivity. In contrast, trained athletes store IMCL to a similar level as insulinresistant individuals, while being highly insulin sensitive; a phenomenon referred to as the 'athlete's paradox' [4]. More recently, however, size, number, subcellular distribution and mitochondrial tethering of lipid droplets, as well as their decoration with lipid droplet coat proteins, appear to be 
discriminating determinants of fat oxidative capacity in an insulin sensitivity-dependent fashion $[5,6]$.

The aim of this review is to summarise recent insights into exercise-mediated changes in lipid metabolism and insulin sensitivity in relation to lipid droplet characteristics in human liver and muscle.

\section{Effects of acute exercise on lipid metabolism in human skeletal muscle}

IMCL utilisation during endurance exercise Stable isotope measurements in combination with muscle biopsies taken before and after exercise give insights in substrate use during exercise. Both, individuals with type 2 diabetes and obese control participants mainly rely on fatty acids originating from the circulation $[1,7]$. Additionally, compared with endurancetrained athletes, individuals with type 2 diabetes and obese individuals use very little IMCL as an energy source [1, 8] (Fig. 1). The lower contribution of IMCL to total fat oxidation in individuals with type 2 diabetes patients, as compared with trained individuals, may originate from dysfunctional adipose tissue and concomitant elevated plasma NEFA levels [7]. This notion is substantiated by the observation that upon acute administration of acipimox, a plasma lipid-lowering agent, the contribution of IMCL to total fat oxidation increases in type 2 diabetes patients [9]. On the other hand, it has also been observed that the contribution of IMCL to total fat oxidation was higher in trained athletes vs individuals with type 2 diabetes when matched for plasma NEFA levels [1]. This suggests that liberation of fatty acid from myocellular lipid droplets in individuals with type 2 diabetes is compromised relative to trained athletes (Fig. 1).

Myocellular lipid droplets are viewed as dynamic organelles that store and release fatty acids upon changes in energy demand and supply [10]. Lipid droplet characteristics, such as number, size, location and protein decoration, are determinants of insulin resistance $[5,11]$ and are remarkably different between athletes and individuals with type 2 diabetes. Unlike athletes, those with type 2 diabetes store more lipid droplets in the subsarcolemmal region $[5,8,11]$ in glycolytic type II muscle fibres [5]. Lipid droplet coating proteins of the perilipin (PLIN) family play a role in lipid-droplet turnover by interacting with lipases, such as adipose triglyceride lipase (ATGL) and hormone sensitive lipase (HSL), and their coactivators. PLIN2, PLIN3 and PLIN5 are the main PLINs present in human skeletal muscle [10]. PLIN2 negatively regulates ATGL-mediated lipid droplet lipolysis by hindering access of ATGL to the lipid droplet surface [12]. PLIN3 coats nascent lipid droplets and associates with fat oxidation rates [13]. PLIN5 regulates lipolytic rate in an energy demanddependent fashion to match fatty acid release from lipid droplets with mitochondrial fatty acid oxidation [10]. While acute exercise does not affect total PLIN5 or ATGL content [1], redistribution of PLIN5 and ATGL upon exercise to match the acute changes in energy demand may occur. Examination of the subcellular redistribution of proteins involved in myocellular lipid droplet lipolysis upon exercise has recently become possible at the level of individual lipid droplets via advanced imaging [10]. Thus, it has been shown that healthy lean participants preferentially use lipid droplets coated with PLIN2 [14, 15] and PLIN5 [14] during endurance exercise. Interestingly, the number of PLIN5-coated lipid droplets in endurance-trained athletes is higher than in individuals type 2 diabetes [6]. In addition, we observed that people with type 2 diabetes have a higher myocellular PLIN2 protein content than endurance-trained athletes [5]. Although it is commonly accepted that PLIN2 that is not bound to the lipid droplet surface is ubiquitinated and targeted for degradation, it has not yet been proven that the higher PLIN2 content in the muscle of type 2 diabetic individuals indeed implies increased decoration of the lipid droplet surface with PLIN2. Taken together, this indicates that the muscle of endurance-trained athletes is equipped for a higher exercise-mediated lipid-droplet turnover than that of individuals with type 2 diabetes. In addition, the site of lipid storage, with athletes having more lipid droplets in the intramyofibrillar area than individuals with type 2 diabetes, spatially and functionally matches a high lipid dropletderived fat oxidative capacity. Indeed, reduction in lipid droplet number and content in the intramyofibrillar area upon acute exercise is observed [8, 16], suggesting a preferential utilisation of intramyofibrillar lipid droplets during exercise.

These studies provide novel and important insights on lipid droplet utilisation in relation to their location and protein decoration and give a better understanding of how lipiddroplet turnover is regulated during exercise in healthy individuals. This type of data, however, is lacking in individuals with type 2 diabetes. For full comprehension of why lipid droplet utilisation is compromised during endurance exercise in individuals with type 2 diabetes, a tracer study to make the distinction between whether plasma or lipid droplet-derived fatty acids are used for oxidation, along with lipid dropletspecific analysis of lipid droplet coat proteins and analysis of lipid droplet location, should be performed pre- and postendurance exercise in individuals with type 2 diabetes.

Lipid droplet-mitochondria interaction during endurance exercise The more pronounced utilisation of intramyofibrillar lipid droplets during exercise may well be related to the observation that, in skeletal muscle, most lipid droplets (predominantly in the trained state, in the intramyofibrillar area) are in close proximity to mitochondria [17-19]. At the interaction sites of mitochondria and lipid droplets, there is an abundance of PLIN5 [18]. In line with the role of PLIN5 in matching lipolytic rate to fatty acid oxidation rate, PLIN5 may play a 

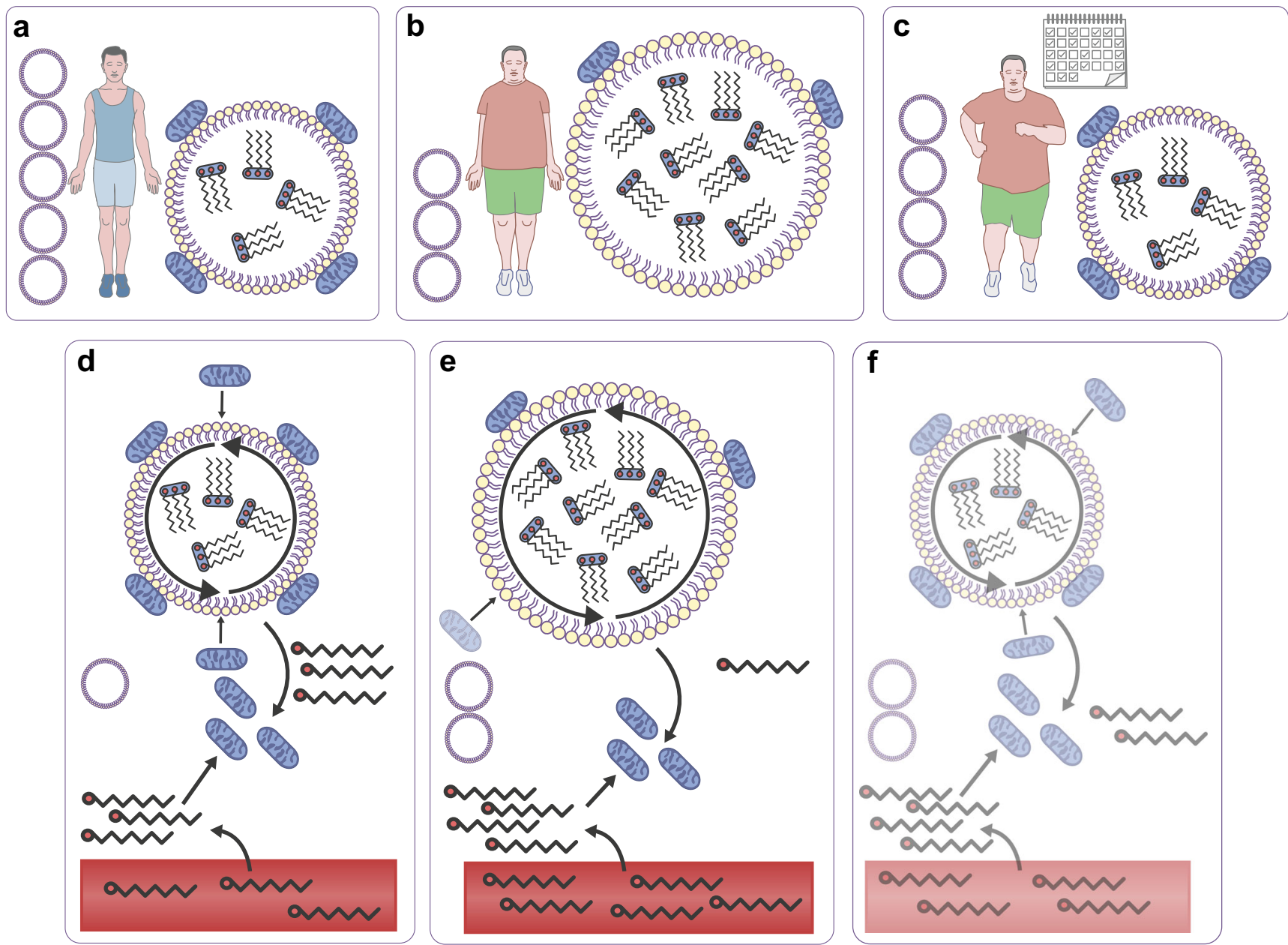

Fig. 1 Skeletal muscle lipid metabolism: acute exercise and endurance training effects. Healthy active/endurance-trained athletes have IMCL content stored in many small lipid droplets (a). Contrarily, in people who are metabolically compromised (i.e. obese and type 2 diabetic individuals), the same amount of IMCL is stored in fewer, but larger lipid droplets (b). Triacylglycerols are shown within the lipid droplets. Lipid droplet number is depicted by the stacked circles next to the image of the lean/obese individuals. Lipid droplet-mitochondria interaction is higher in athletes vs metabolically compromised individuals. Upon enduranceexercise intervention (training depicted by the calendar), lipid droplet morphology and lipid droplet-mitochondria interactions changes towards the athlete-like phenotype in individuals who are metabolically compromised (c). (d, e) During an acute endurance exercise bout, fatty acids originating from lipid droplets, as well as from the circulation are used as an energy source. Endurance-trained athletes rely more heavily on IMCL to fuel exercise and have a higher lipid-droplet turnover (i.e. storage of circulation-derived fatty acids in lipid droplets and release of fatty acids originating from lipid droplets for fatty acid oxidation) than those who are metabolically compromised. This reduces the number of lipid droplets, as depicted by a smaller stack of lipid droplets in (d) vs (e). The interaction between lipid droplets and mitochondria is higher in endurance-trained athletes. This may facilitate fatty acid oxidation during exercise. Changes that occur upon exercise training in metabolically compromised individuals are shown in (b) and (c), i.e. an increased lipid droplet-mitochondrial interaction, and smaller and more lipid droplets. The hypothesised changes upon an acute exercise bout after metabolically compromised individuals have followed an endurance training intervention are represented in (e) and (f): lipid droplet-mitochondrial interaction is anticipated to increase during exercise, and lipid turnover and IMCL utilisation starts to mimic the events in athletes. Hypothetical changes are depicted using transparent illustrations. This figure is available as part of a downloadable slideset role in shuttling or chaperoning lipid droplet-released fatty acids to mitochondria for oxidation [18]. Recent studies have suggested that, when interacting with lipid droplets, mitochondria have different cellular functions than non-lipiddroplet-interacting mitochondria [19, 20]. For skeletal muscle, it has been suggested that mitochondria that are in contact with lipid droplets have a greater capacity for ATP production than non-lipid-droplet-interacting mitochondria [19]. Thus, lipid droplet-mitochondrial tethering may facilitate high fat oxidation by liberating fatty acids in the direct vicinity of mitochondria with a high capacity to oxidise fatty acids, thereby contributing to ATP maintenance during exercise. At present, experimental proof in humans for these functional processes is lacking. It should be noted, though, that trained individuals possess higher PLIN5 levels, have more PLIN5coated lipid droplets [6] and may, thus, have more lipid droplet-mitochondrial interaction sites than individuals with type 2 diabetes. Lipid droplet-mitochondria interactions are 
not different between healthy lean and healthy obese participants [21, 22], but these data are lacking for individuals with type 2 diabetes in comparison with endurance-trained athletes.

Data on changes in lipid droplet-mitochondria tethering during exercise are only available for endurance-trained athletes. In male elite cross-country skiers, lipid dropletmitochondria interactions increase upon an acute exercise bout despite unaltered IMCL content [16]. In endurancetrained women, lipid droplet-mitochondria tethering increases during exercise, with a concomitant reduction in IMCL content [23]. The latter study suggests that lipid droplet-mitochondrial interaction upon exercise promotes fatty acid oxidation. The seemingly contradictory finding that an exercise-mediated increase in lipid dropletmitochondria interaction is paralleled by reduced IMCL content in women [23] but not in men [16] might originate from sex differences, as reviewed recently [24]. A lack of a reduction in IMCL upon exercise (as observed in the male elite cross-country skiers) may also be reflective of a high IMCL turnover (IMCL utilisation during exercise matches fatty acid incorporation into lipid droplets). The underlying mechanism for increased mitochondria-lipid droplet tethering during exercise and whether PLIN5 is important for the capacity to increase lipid droplet-mitochondrial tethering are so far unknown. Furthermore, it is not clear whether lipid droplet-mitochondrial tethering is disturbed in individuals with type 2 diabetes. The literature indicates that PLIN5 is important for lipid droplet-mitochondrial tethering $[18,20]$ in oxidative tissues. PLIN5 protein quantification in individual lipid droplets should be performed concomitantly with lipid droplet-mitochondrial interaction analyses in athletes and in those with type 2 diabetes upon an acute exercise bout to gain a better understanding of how lipid droplet-mitochondrial tethering works and if the capacity to tether additional mitochondria to lipid droplets upon exercise is compromised in individuals with type 2 diabetes (Fig. 1).

\section{Effects of exercise training on lipid metabolism in human skeletal muscle}

Mitochondrial respiratory capacity Compromised mitochondrial respiratory capacity is frequently reported in type 2 diabetes [25-27] and obesity [26], albeit not always confirmed [28]. A potent way to increase mitochondrial respiratory capacity and a concomitant increase in fat oxidation is endurance training. Several studies have shown that mitochondrial respiratory capacity and fat oxidation increases upon endurance exercise training, even in type 2 diabetic $[25,29]$ and obese [25, 30] participants.

IMCL storage, lipid droplet morphology and lipid dropletmitochondria interactions As well as increasing mitochondrial capacity, endurance training also is an effective intervention to improve fat oxidation and modulate fat storage in the skeletal muscle of lean sedentary participants [31]. Several studies have shown that endurance training (4-16 weeks) may affect lipid droplet characteristics without major changes in total IMCL content in type 2 diabetic [5, 11, 25, 29, 32], obese $[21,25,33]$, and healthy lean, sedentary $[21,34,35]$ participants. In most of these studies, however, insulin sensitivity improved. To understand this seemingly paradoxical observation, we need to focus on what happens at the lipid droplet level, rather than at the total IMCL content level. Upon exercise training, lipid droplet size $[5,22,32]$ and subsarcolemmal lipid droplet content $[11,21,22]$ reduces, while intramyofibrillar lipid droplet content increases [22]. These exercise-mediated changes, in previously untrained insulinresistant individuals, resembles the IMCL storage pattern observed in insulin-sensitive endurance-trained athletes. This 'athlete-like lipid droplet phenotype' is characterised by many small lipid droplets in the intramyofibrillar region in type I muscle fibres with an abundance of PLIN5, and tethering of PLIN5-positive lipid droplets to mitochondria. In contrast, in individuals with type 2 diabetes, fewer but larger lipid droplets

\section{Summary of acute exercise effects on myocellular lipid droplets and mitochondrial interaction}

1 Athletes rely more on IMCL during aerobic exercise than individuals with type 2 diabetes

Individuals with type 2 diabetes rely more on fatty acids from the circulation during exercise than athletes

Muscle of athletes is well-equipped for higher exercise-mediated lipid droplet

3 turnover based on having a higher number of PLIN5-coated lipid droplets than individuals with type 2 diabetes

In muscle of athletes, lipid droplet-mitochondria tethering is increased upon a single bout of exercise 
are observed, with a higher fraction of lipid droplets in the subsarcolemmal region of type II muscle fibres [5]. Lipid droplet-mitochondrial tethering increases upon endurance training in obese participants [21,22], while no such effect was observed in individuals with type 2 diabetes [36]. All of these athlete-like changes were observed in training programmes that were carried out for more than 10 weeks (Fig. 1). Short-term training (4 weeks) in obese participants did not change lipid droplet size and number, but lipid droplet-mitochondrial interaction was increased [33]. This indicates that an athlete-like shift in lipid droplet phenotype permits storage of IMCL without impeding insulin sensitivity. A training-induced improvement in lipid dropletmitochondrial tethering appears to be an early adaptation of endurance training that is crucial for remodelling of the IMCL storage pattern.

IMCL utilisation/lipid-droplet turnover during endurance exercise Training studies in healthy lean participants show that endurance training for 6 weeks promotes IMCL utilisation during exercise [14, 35, 37]. While in the untrained state PLIN2- and PLIN5-coated lipid droplets are preferentially used during exercise, 6 weeks of endurance training resulted in preferred utilisation of PLIN5-coated lipid droplets during exercise [14]. While the effect of exercise training on proteins involved in lipid-droplet turnover, such as PLIN2, PLIN5 and ATGL, has been measured, data on the effect of endurance training on IMCL utilisation and lipid-droplet turnover during an exercise bout in obese participants and individuals with type 2 diabetes is lacking (Fig. 1f). PLIN5 gene expression and protein content upon an endurance training intervention increases in obese participants and individuals with type 2 diabetes [5, 33, 38, 39]. For PLIN2 [5, 33, 38-40], PLIN3 $[5,33,38]$ and ATGL $[5,38]$ the training effects are less consistent, either showing an increase or no change in the general population. Increased PLIN5 protein content upon endurance training indicates that IMCL use during exercise is facilitated and that lipolysis rates of lipid droplets are better matched to mitochondrial fatty acid oxidation rates in individuals with type 2 diabetes vs baseline. To test these mechanisms in a human setting, acute exercise studies in participants with type 2 diabetes are needed and should include fatty acid tracers and muscle biopsies to study IMCL utilisation during exercise, and changes in PLIN5 protein content at the lipid droplet surface before and after training. Additionally, in vitro studies in human primary myotubes obtained from endurancetrained athletes and individuals with type 2 diabetes, in combination with imaging of fatty acid tracers with live-cell imaging, can give important insights into turnover of individual lipid droplets upon exposure to different stimuli resembling exercise. Moreover, to study the direct role of PLIN5 in lipiddroplet turnover, these in vitro studies should be combined with overexpression of fluorescently tagged PLIN5 to test whether PLIN5-coated lipid droplets indeed have a higher lipid-droplet turnover.

\section{Exercise training and nutritional state: training in the fasted state}

In most of the studies discussed above, the timing of meal intake relative to the training sessions was not monitored strictly or intentionally timed so that participants trained fasted. Interestingly, training in the (overnight) fasted state has gained popularity to promote fat oxidative capacity. Upon fasting, adipose tissue lipolysis and plasma NEFA levels increase. The increase in NEFA drives myocellular uptake of fatty acids and, thus, can promote IMCL storage and oxidation of fatty acids. Indeed, fat oxidation rates during acute exercise in the fasted state are higher than in the fed state $[41,42]$. Also, the (sustained) increase in NEFA levels upon exercise in the fasted state can hypothetically provide ligands

\begin{tabular}{|l|}
\hline Summary of the effects of endurance training on myocellular lipid droplets \\
\hline 1 IMCL lipid storage pattern in individuals with type 2 diabetes changes towards an \\
athlete-like phenotype upon endurance training \\
Lipid droplet-mitochondria tethering increases upon endurance training in obese \\
participants. Until now, this has not been reported for individuals with type 2 \\
diabetes \\
(3) Endurance training increases IMCL utilisation during exercise in healthy lean \\
untrained participants \\
Acute exercise studies including muscle biopsies and tracers in individuals with type \\
2 diabetes before and after a training intervention are needed to study the effects \\
of training on IMCL use and lipid droplet turnover during exercise
\end{tabular}




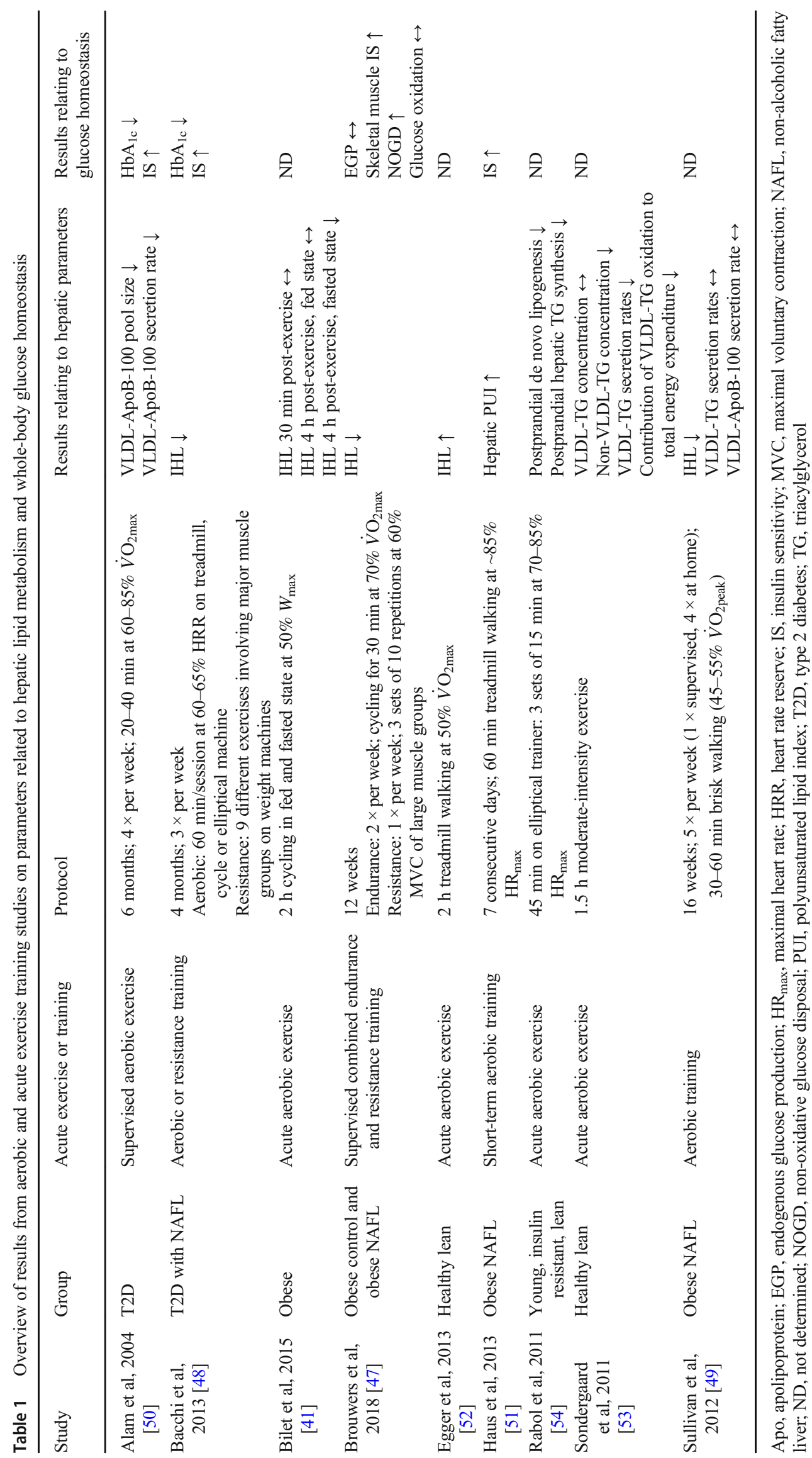




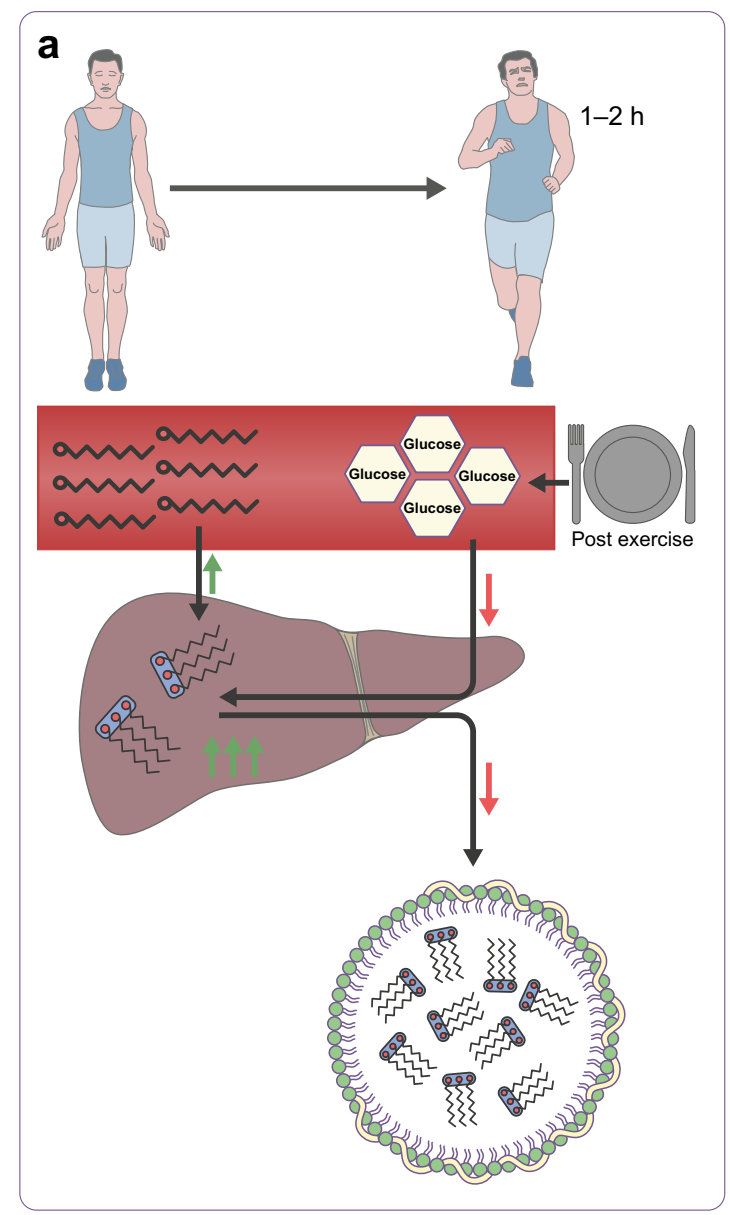

Fig. 2 Liver lipid metabolism: acute exercise and endurance training effects. IHL content is lower in healthy lean individuals than in those who are metabolically compromised. This may be a consequence of lower plasma NEFA levels and lower rates of de novo lipogenesis in lean vs metabolically compromised individuals. (a) Upon acute endurance exercise, especially in the fasted state, IHL content rises, most likely due to increased plasma NEFA levels. Furthermore, VLDLtriacylglycerol secretion rates drop during acute exercise, and de novo lipogenesis is blunted due to higher postprandial glycogen synthesis by the muscle, thereby reducing glucose availability for lipid synthesis by the

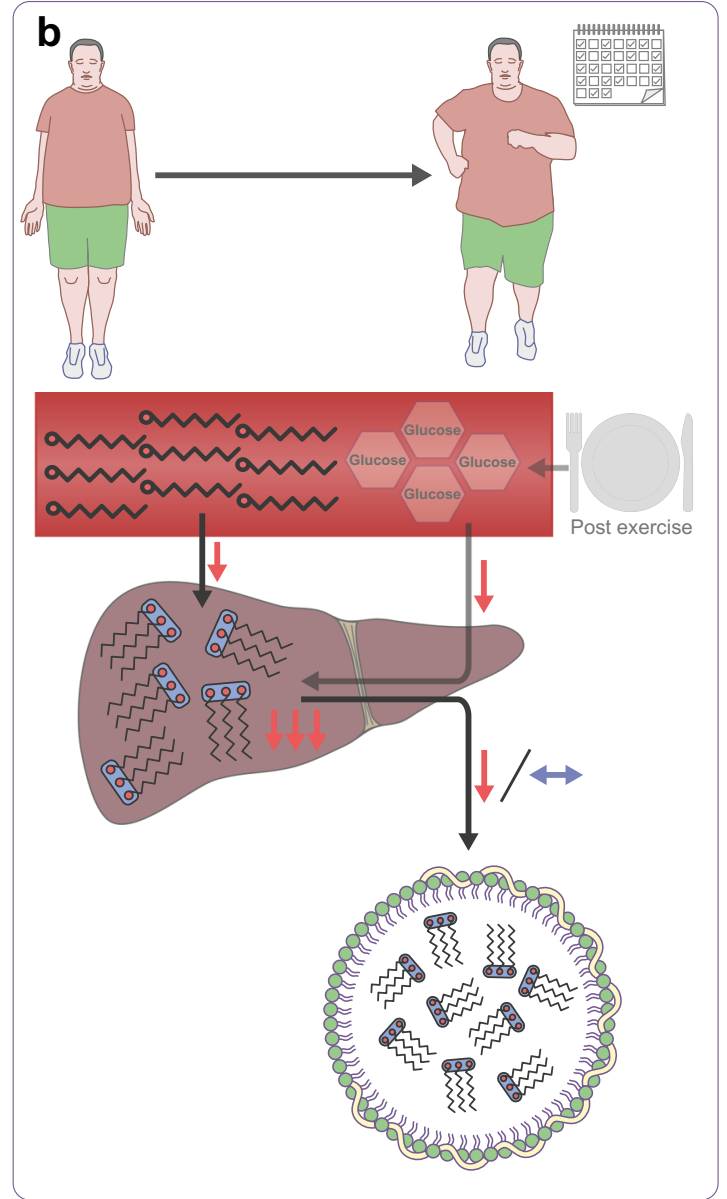

liver. (b) The underlying mechanisms that are (hypothetically) involved during endurance training in metabolically compromised individuals are shown (exercise training depicted by the calendar); these include reduced de novo lipogenesis, and improved postprandial glucose and NEFA uptake by the muscle and, thus, lower availability of glucose and NEFA for the liver to synthesise lipids. In addition, VLDL-triacylglycerol secretion rate upon endurance training in metabolically compromised individuals drops or is unchanged. Hypothetical changes are depicted using transparent illustrations. This figure is available as part of a downloadable slideset for peroxisome proliferator-activated receptor (PPAR)-mediated gene expression and, thereby, promote an adaptive response in regard to fat metabolism. Interestingly, endurance training in the fasted state improves glucose tolerance to a greater extent than training in the fed state [43]. Data on functional adaptations (like increased fat oxidative capacity) following training in the fasted state are inconsistent $[35,37,44,45]$. Acute exercise studies measuring IMCL utilisation (with fatty acid tracers and in muscle biopsies) have been performed in the fasted state and show IMCL utilisation during exercise $[1,14,15]$. Compared with exercise in the fed state, exercising in the fasted state results in higher NEFA levels, higher fat oxidation rates and a drop in IMCL content [42]. We previously observed that, over a wide range of interventions, elevated plasma fatty acids promote IMCL storage. Whether this also occurs during exercise in the fasted state and translates into a higher flux of fatty acids in lipid droplets during exercise remains to be studied.

Upon 6 weeks of endurance training, IMCL content drops during a single exercise bout in the fasted state. This drop in IMCL content upon acute exercise was similar if the training was performed in the carbohydrate-fed state vs that fasted state $[35,37]$. Currently, most training interventions under fasted conditions have only been performed in healthy lean participants and translation towards the type 2 diabetes population should be done carefully. Based on the results in healthy lean individuals, training while fasted may induce more IMCL remodelling due to a higher stimulus for lipid-droplet turnover in individuals with type 2 diabetes. Before drawing these 


\section{Summary of the effects of nutritional state on lipid metabolism during exercise}

Fat oxidation rates and NEFA levels are higher during exercise in the fasted state than in the fed state

Endurance training in the fasted state improves glucose tolerance to a greater extent than training in the fed state

Currently, most training interventions under fasted conditions have been performed in healthy lean participants and translation towards the type 2 diabetes population should, hence, be done carefully

conclusions, training interventions in the fasted vs fed state should be performed in individuals with type 2 diabetes.

\section{Exercise and liver lipid metabolism}

Intrahepatic lipid (IHL) storage is associated with type 2 diabetes and cardiovascular diseases. The poor accessibility of the liver in healthy individuals means that most studies towards the effect of acute exercise and exercise training on IHLs and lipid metabolism in humans are based upon non-invasive techniques, such as MRI and tracer studies. These studies have revealed that diet and exercise both affect IHL content in obese patients with a non-alcoholic fatty liver and/or type 2 diabetes [46]. Upon endurance training for 12 weeks to 4 months, IHL content is reduced [47-49]; this has recently been extensively reviewed in Diabetologia [46]. Reductions in IHL content has been shown to occur in parallel with improvements in wholebody/muscle insulin sensitivity $[47,48]$, however, hepatic insulin sensitivity was unaffected [47] (Table 1). While a drop in IHL levels after endurance training generally occurs in the absence of changes in body weight, we observed that the training-mediated drop in IHL correlated with a drop in body fat mass $[46,47]$. Increased IHL storage is, in general, not associated with disturbed VLDL-triacylglycerol secretion rates [46], and data on VLDL(-triacylglycerol) secretion rates upon endurance training is contradictory, either showing no change [49] or a decrease [50] (Table 1). It is tempting to speculate that exercise-mediated improvements in whole-body insulin sensitivity include reduced de novo lipogenesis in the liver, thereby contributing to a lower IHL content. While we are not aware of any studies underpinning this notion, it is interesting to note that a short-term (7 day) training programme resulted in altered composition (but not content) of IHL. After training, IHL contained more polyunsaturated fatty acids [51]; this is in line with lower de novo lipogenesis, which gives rise to saturated fat (Fig. 2, Table 1).

As exercise training reduces IHL content [47, 48], one could suggest that IHL also drops upon acute exercise. We observed that, upon $2 \mathrm{~h}$ of endurance exercise, IHL content was unaffected, irrespective of participants being in the fed or fasted stated. After exercise and upon recovery in the fasted state, however, we observed an increase in IHL [41]. Additionally, IHL increases upon an exercise bout in active lean participants who consumed a light meal before the start of the exercise [52]. Interestingly, in both studies [41, 52],

\section{Summary of the effects of exercise on hepatic lipid metabolism}

1.

Aerobic exercise training reduces IHL content in metabolically compromised populations concomitantly with improving insulin sensitivity

2 Aerobic exercise training does not directly affect VLDL-triacylglycerol secretion rates

3 Short-term aerobic exercise training increases polyunsaturated fatty acid content of IHLs; this is compatible with reduced de novo lipogenesis

4 Acute exercise increases IHL content in healthy lean and obese participants

5 Acute exercise reduces postprandial hepatic de novo lipogenesis and triacylglycerol synthesis in lean insulin-resistant individuals 
increased IHL content after exercise occurred in the presence of elevated plasma NEFA levels. If this rise in plasma NEFAs is prevented by providing a glucose drink every half hour during and after exercise, IHL does not increase. This indicates that the rise in plasma NEFA levels upon exercise drives the increased IHL content after an exercise bout.

IHL can be used during exercise, upon secretion of VLDLtriacylglycerols into the bloodstream. VLDL-triacylglycerol kinetic analyses during an acute exercise bout in the fasted state show that VLDL-triacylglycerol secretion rates drop during exercise and that the contribution of these particles to total energy expenditure is decreased [53]. Thus, besides the increase in NEFA influx, the lower VLDL-triacylglycerol secretion rates during exercise may also contribute to the increase in IHL content after acute exercise in the fasted state (Fig. 2). In lean, normoglycaemic but insulin-resistant individuals, postprandial IHL synthesis and de novo lipogenesis is lower after a single bout of exercise compared with rest [54].

Overall, IHL may increase upon acute exercise, but is lower after training, possibly due to lower postprandial de novo lipogenesis during recovery. It is also lower in endurance-trained individuals. It is currently unknown how the apparent increase in IHL after acute exercise turns into reduced IHL content after endurance training. We cannot exclude that training, per se, is not the major determinant of IHL but that the dietary habits of trained individuals may also make an important contribution.

\section{Concluding remarks}

IMCL and IHL content are increased, and fat oxidative capacity decreased in metabolically compromised individuals, such as obese individuals and those with type 2 diabetes. While endurance exercise training reduces total intracellular fat content in the liver, the effects in muscle indicate remodelling rather than lowering of the myocellular lipid droplet pool. In fact, in most populations and under most conditions, endurance exercise training augments IMCL content. Thus, the ability of exercise to modulate lipid droplet dynamics in the liver and muscle contributes to differences in fat oxidative metabolism. Endurance training in individuals with type 2 diabetes remodels IMCL content towards an athlete-like phenotype, while IHL content is reduced. While many training intervention studies have been performed in metabolically compromised individuals, the effects of acute exercise have not been extensively studied, particularly not in participants with type 2 diabetes. Thus, it is unclear why IMCL utilisation during exercise is lower in individuals with type 2 diabetes and whether the observed IMCL remodelling towards the athlete-like phenotype in these individuals also translates into the anticipated increase in IMCL utilisation during exercise. Study findings on the effects of sex differences and exercise intensity on IMCL use during exercise or lipid droplet remodelling upon training are either contradictory or lacking. Compared with skeletal muscle, the underlying mechanisms of the effects of exercise and training on IHL are even more poorly understood. The reduction in IHL content upon training that is observed in metabolically compromised individuals may partly originate from reduced postprandial de novo lipogenesis. Since diurnal rhythms are present in lipid metabolism, future studies should also focus on the effect of timing of exercise on the parameters discussed in this review in order to elucidate the optimal conditions for exercise-induced improvements in insulin sensitivity in individuals with type 2 diabetes.

Funding This work received no specific grant from any funding agency in the public, commercial or not-for-profit sectors.

Author's relationships and activities The authors declare that there are no relationships or activities that might bias, or be perceived to bias, their work.

Contribution statement All authors were responsible for drafting the article and revising it critically for important intellectual content. All authors approved the version to be published.

Open Access This article is licensed under a Creative Commons Attribution 4.0 International License, which permits use, sharing, adaptation, distribution and reproduction in any medium or format, as long as you give appropriate credit to the original author(s) and the source, provide a link to the Creative Commons licence, and indicate if changes were made. The images or other third party material in this article are included in the article's Creative Commons licence, unless indicated otherwise in a credit line to the material. If material is not included in the article's Creative Commons licence and your intended use is not permitted by statutory regulation or exceeds the permitted use, you will need to obtain permission directly from the copyright holder. To view a copy of this licence, visit http://creativecommons.org/licenses/by/4.0/.

\section{References}

1. Bergman BC, Perreault L, Strauss A et al (2018) Intramuscular triglyceride synthesis: importance in muscle lipid partitioning in humans. Am J Physiol Endocrinol Metab 314(2):E152-E164. https://doi.org/10.1152/ajpendo.00142.2017

2. Kiens B (2006) Skeletal muscle lipid metabolism in exercise and insulin resistance. Physiol Rev 86(1):205-243. https://doi.org/10. 1152/physrev.00023.2004

3. van Loon LJ, Greenhaff PL, Constantin-Teodosiu D, Saris WH, Wagenmakers AJ (2001) The effects of increasing exercise intensity on muscle fuel utilisation in humans. J Physiol 536(1):295304. https://doi.org/10.1111/j.1469-7793.2001.00295.x

4. Goodpaster BH, He J, Watkins S, Kelley DE (2001) Skeletal muscle lipid content and insulin resistance: evidence for a paradox in endurance-trained athletes. J Clin Endocrinol Metab 86(12): 5755-5761. https://doi.org/10.1210/jcem.86.12.8075

5. Daemen S, Gemmink A, Brouwers B et al (2018) Distinct lipid droplet characteristics and distribution unmask the apparent contradiction of the athlete's paradox. Mol Metab 17:71-81. https://doi. org/10.1016/j.molmet.2018.08.004 
6. Gemmink A, Daemen S, Brouwers B et al (2018) Dissociation of intramyocellular lipid storage and insulin resistance in trained athletes and type 2 diabetes patients; involvement of perilipin 5? J Physiol 596(5):857-868. https://doi.org/10.1113/JP275182

7. Boon H, Blaak EE, Saris WH, Keizer HA, Wagenmakers AJ, van Loon LJ (2007) Substrate source utilisation in long-term diagnosed type 2 diabetes patients at rest, and during exercise and subsequent recovery. Diabetologia 50(1):103-112. https://doi.org/10.1007/ s00125-006-0482-2

8. Chee C, Shannon CE, Burns A et al (2016) Relative contribution of intramyocellular lipid to whole-body fat oxidation is reduced with age but subsarcolemmal lipid accumulation and insulin resistance are only associated with overweight individuals. Diabetes 65(4): 840-850. https://doi.org/10.2337/db15-1383

9. van Loon LJ, Manders RJ, Koopman R et al (2005) Inhibition of adipose tissue lipolysis increases intramuscular lipid use in type 2 diabetic patients. Diabetologia 48(10):2097-2107. https://doi.org/ 10.1007/s00125-005-1889-x

10. Gemmink A, Goodpaster BH, Schrauwen P, Hesselink MKC (2017) Intramyocellular lipid droplets and insulin sensitivity, the human perspective. Biochim Biophys Acta Mol Cell Biol Lipids 1862(10 Pt B):1242-1249. https://doi.org/10.1016/j.bbalip.2017. 07.010

11. Nielsen J, Mogensen M, Vind BF et al (2010) Increased subsarcolemmal lipids in type 2 diabetes: effect of training on localization of lipids, mitochondria, and glycogen in sedentary human skeletal muscle. Am J Physiol Endocrinol Metab 298(3): E706-E713. https://doi.org/10.1152/ajpendo.00692.2009

12. Feng YZ, Lund J, Li Y et al (2017) Loss of perilipin 2 in cultured myotubes enhances lipolysis and redirects the metabolic energy balance from glucose oxidation towards fatty acid oxidation. J Lipid Res 58(11):2147-2161. https://doi.org/10.1194/jlr.M079764

13. Covington JD, Noland RC, Hebert RC et al (2015) Perilipin 3 differentially regulates skeletal muscle lipid oxidation in active, sedentary and type 2 diabetic males. J Clin Endocrinol Metab 100(10):3683-3692. https://doi.org/10.1210/JC.2014-4125

14. Shepherd SO, Cocks M, Tipton KD et al (2013) Sprint interval and traditional endurance training increase net intramuscular triglyceride breakdown and expression of perilipin 2 and 5. J Physiol 591(3):657-675. https://doi.org/10.1113/jphysiol.2012.240952

15. Shepherd SO, Cocks M, Tipton KD et al (2012) Preferential utilization of perilipin 2-associated intramuscular triglycerides during $1 \mathrm{~h}$ of moderate-intensity endurance-type exercise. Exp Physiol 97(8):970-980. https://doi.org/10.1113/expphysiol.2012.064592

16. Koh HE, Nielsen J, Saltin B, Holmberg HC, Ortenblad N (2017) Pronounced limb and fibre type differences in subcellular lipid droplet content and distribution in elite skiers before and after exhaustive exercise. J Physiol 595(17):5781-5795. https://doi.org/ $10.1113 / \mathrm{JP} 274462$

17. Shaw CS, Jones DA, Wagenmakers AJ (2008) Network distribution of mitochondria and lipid droplets in human muscle fibres. Histochem Cell Biol 129(1):65-72. https://doi.org/10.1007/ s00418-007-0349-8

18. Gemmink A, Daemen S, Kuijpers HJH et al (2018) Superresolution microscopy localizes perilipin 5 at lipid dropletmitochondria interaction sites and at lipid droplets juxtaposing to perilipin 2. Biochim Biophys Acta Mol Cell Biol Lipids 1863(11): 1423-1432. https://doi.org/10.1016/j.bbalip.2018.08.016

19. Bleck CKE, Kim Y, Willingham TB, Glancy B (2018) Subcellular connectomic analyses of energy networks in striated muscle. Nat Commun 9(1):5111. https://doi.org/10.1038/s41467-018-07676-y

20. Benador IY, Veliova M, Mahdaviani K et al (2018) Mitochondria bound to lipid droplets have unique bioenergetics, composition, and dynamics that support lipid droplet expansion. Cell Metab 27(4): 869-885. https://doi.org/10.1016/j.cmet.2018.03.003
21. Devries MC, Samjoo IA, Hamadeh MJ et al (2013) Endurance training modulates intramyocellular lipid compartmentalization and morphology in skeletal muscle of lean and obese women. $\mathrm{J}$ Clin Endocrinol Metab 98(12):4852-4862. https://doi.org/10. 1210/jc.2013-2044

22. Samjoo IA, Safdar A, Hamadeh MJ et al (2013) Markers of skeletal muscle mitochondrial function and lipid accumulation are moderately associated with the homeostasis model assessment index of insulin resistance in obese men. PLoS One 8(6):e66322. https://doi. org/10.1371/journal.pone.0066322

23. Devries MC, Lowther SA, Glover AW, Hamadeh MJ, Tarnopolsky MA (2007) IMCL area density, but not IMCL utilization, is higher in women during moderate-intensity endurance exercise, compared with men. Am J Physiol Regul Integr Comp Physiol 293(6): R2336-R2342. https://doi.org/10.1152/ajpregu.00510.2007

24. Devries MC (2016) Sex-based differences in endurance exercise muscle metabolism: impact on exercise and nutritional strategies to optimize health and performance in women. Exp Physiol 101(2):243-249. https://doi.org/10.1113/EP085369

25. Meex RC, Schrauwen-Hinderling VB, Moonen-Kornips E et al (2010) Restoration of muscle mitochondrial function and metabolic flexibility in type 2 diabetes by exercise training is paralleled by increased myocellular fat storage and improved insulin sensitivity. Diabetes 59(3):572-579. https://doi.org/10.2337/db09-1322

26. Kelley DE, He J, Menshikova EV, Ritov VB (2002) Dysfunction of mitochondria in human skeletal muscle in type 2 diabetes. Diabetes 51(10):2944-2950. https://doi.org/10.2337/diabetes.51.10.2944

27. Phielix E, Schrauwen-Hinderling VB, Mensink M et al (2008) Lower intrinsic ADP-stimulated mitochondrial respiration underlies in vivo mitochondrial dysfunction in muscle of male type 2 diabetic patients. Diabetes 57(11):2943-2949. https://doi.org/10. 2337/db08-0391

28. De Feyter HM, van den Broek NM, Praet SF, Nicolay K, van Loon LJ, Prompers JJ (2008) Early or advanced stage type 2 diabetes is not accompanied by in vivo skeletal muscle mitochondrial dysfunction. Eur J Endocrinol 158(5):643-653. https://doi.org/10.1530/eje07-0756

29. Pino MF, Stephens NA, Eroshkin AM et al (2019) Endurance training remodels skeletal muscle phospholipid composition and increases intrinsic mitochondrial respiration in men with type 2 diabetes. Physiol Genomics 51(11):586-595. https://doi.org/10. 1152/physiolgenomics.00014.2019

30. Bruce CR, Thrush AB, Mertz VA et al (2006) Endurance training in obese humans improves glucose tolerance and mitochondrial fatty acid oxidation and alters muscle lipid content. Am J Physiol Endocrinol Metab 291(1):E99-E107. https://doi.org/10.1152/ ajpendo.00587.2005

31. Schrauwen P, van Aggel-Leijssen DP, Hul G et al (2002) The effect of a 3-month low-intensity endurance training program on fat oxidation and acetyl-CoA carboxylase-2 expression. Diabetes 51(7):2220-2226. https://doi.org/10.2337/diabetes.51.7.2220

32. He J, Goodpaster BH, Kelley DE (2004) Effects of weight loss and physical activity on muscle lipid content and droplet size. Obes Res 12(5):761-769. https://doi.org/10.1038/oby.2004.92

33. Shepherd SO, Cocks M, Meikle PJ et al (2017) Lipid droplet remodelling and reduced muscle ceramides following sprint interval and moderate-intensity continuous exercise training in obese males. Int J Obes 41(12):1745-1754. https://doi.org/10.1038/ijo. 2017.170

34. Tarnopolsky MA, Rennie CD, Robertshaw HA, FedakTarnopolsky SN, Devries MC, Hamadeh MJ (2007) Influence of endurance exercise training and sex on intramyocellular lipid and mitochondrial ultrastructure, substrate use, and mitochondrial enzyme activity. Am J Physiol Regul Integr Comp Physiol 292(3):R1271-R1278. https://doi.org/10.1152/ajpregu.00472. 2006 
35. Van Proeyen K, Szlufcik K, Nielens H et al (2011) High-fat diet overrules the effects of training on fiber-specific intramyocellular lipid utilization during exercise. J Appl Physiol 111(1):108-116. https://doi.org/10.1152/japplphysiol.01459.2010

36. Koh HE, Ortenblad N, Winding KM, Hellsten Y, Mortensen SP, Nielsen J (2018) High-intensity interval, but not endurance, training induces muscle fiber type-specific subsarcolemmal lipid droplet size reduction in type 2 diabetic patients. Am J Physiol Endocrinol Metab 315(5):E872-E884. https://doi.org/10.1152/ ajpendo.00161.2018

37. Van Proeyen K, Szlufcik K, Nielens H, Ramaekers M, Hespel P (2011) Beneficial metabolic adaptations due to endurance exercise training in the fasted state. J Appl Physiol 110(1):236-245. https:// doi.org/10.1152/japplphysiol.00907.2010

38. Louche K, Badin PM, Montastier E et al (2013) Endurance exercise training up-regulates lipolytic proteins and reduces triglyceride content in skeletal muscle of obese subjects. J Clin Endocrinol Metab 98(12):4863-4871. https://doi.org/10.1210/jc.2013-2058

39. Peters SJ, Samjoo IA, Devries MC, Stevic I, Robertshaw HA, Tarnopolsky MA (2012) Perilipin family (PLIN) proteins in human skeletal muscle: the effect of sex, obesity, and endurance training. Appl Physiol Nutr Metab 37(4):724-735. https://doi.org/10.1139/ h2012-059

40. Shaw CS, Shepherd SO, Wagenmakers AJ, Hansen D, Dendale P, van Loon LJ (2012) Prolonged exercise training increases intramuscular lipid content and perilipin 2 expression in type I muscle fibers of patients with type 2 diabetes. Am J Physiol Endocrinol Metab 303(9):E1158-E1165. https://doi.org/10.1152/ajpendo.00272. 2012

41. Bilet L, Brouwers B, van Ewijk PA et al (2015) Acute exercise does not decrease liver fat in men with overweight or NAFLD. Sci Rep 5(1):9709. https://doi.org/10.1038/srep09709

42. De Bock K, Richter EA, Russell AP et al (2005) Exercise in the fasted state facilitates fibre type-specific intramyocellular lipid breakdown and stimulates glycogen resynthesis in humans. J Physiol 564(2):649-660. https://doi.org/10.1113/jphysiol.2005. 083170

43. Van Proeyen K, Szlufcik K, Nielens H et al (2010) Training in the fasted state improves glucose tolerance during fat-rich diet. J Physiol 588(21):4289-4302. https://doi.org/10.1113/jphysiol. 2010.196493

44. Stannard SR, Buckley AJ, Edge JA, Thompson MW (2010) Adaptations to skeletal muscle with endurance exercise training in the acutely fed versus overnight-fasted state. J Sci Med Sport 13(4): 465-469. https://doi.org/10.1016/j.jsams.2010.03.002

45. De Bock K, Derave W, Eijnde BO et al (2008) Effect of training in the fasted state on metabolic responses during exercise with carbohydrate intake. J Appl Physiol 104(4):1045-1055. https:// doi.org/10.1152/japplphysiol.01195.2007

46. Brouwers B, Hesselink MK, Schrauwen P, Schrauwen-Hinderling VB (2016) Effects of exercise training on intrahepatic lipid content in humans. Diabetologia 59(10):2068-2079. https://doi.org/10. 1007/s00125-016-4037-x

47. Brouwers B, Schrauwen-Hinderling VB, Jelenik T et al (2018) Exercise training reduces intrahepatic lipid content in people with and people without nonalcoholic fatty liver. Am J Physiol Endocrinol Metab 314(2):E165-E173. https://doi.org/10.1152/ ajpendo.00266.2017

48. Bacchi E, Negri C, Targher G et al (2013) Both resistance training and aerobic training reduce hepatic fat content in type 2 diabetic subjects with nonalcoholic fatty liver disease (the RAED2 Randomized Trial). Hepatology 58(4):1287-1295. https://doi.org/ 10.1002/hep.26393

49. Sullivan S, Kirk EP, Mittendorfer B, Patterson BW, Klein S (2012) Randomized trial of exercise effect on intrahepatic triglyceride content and lipid kinetics in nonalcoholic fatty liver disease. Hepatology 55(6):1738-1745. https://doi.org/10.1002/hep.25548

50. Alam S, Stolinski M, Pentecost C et al (2004) The effect of a sixmonth exercise program on very low-density lipoprotein apolipoprotein B secretion in type 2 diabetes. J Clin Endocrinol Metab 89(2):688-694. https://doi.org/10.1210/jc.2003-031036

51. Haus JM, Solomon TP, Kelly KR et al (2013) Improved hepatic lipid composition following short-term exercise in nonalcoholic fatty liver disease. J Clin Endocrinol Metab 98(7):E1181-E1188. https://doi.org/10.1210/jc.2013-1229

52. Egger A, Kreis R, Allemann S et al (2013) The effect of aerobic exercise on intrahepatocellular and intramyocellular lipids in healthy subjects. PLoS One 8(8):e70865. https://doi.org/10.1371/ journal.pone. 0070865

53. Sondergaard E, Rahbek I, Sorensen LP et al (2011) Effects of exercise on VLDL-triglyceride oxidation and turnover. Am J Physiol Endocrinol Metab 300(5):E939-E944. https://doi.org/10.1152/ ajpendo.00031.2011

54. Rabol R, Petersen KF, Dufour S, Flannery C, Shulman GI (2011) Reversal of muscle insulin resistance with exercise reduces postprandial hepatic de novo lipogenesis in insulin resistant individuals. Proc Natl Acad Sci U S A 108(33):13705-13709. https://doi.org/ 10.1073/pnas.1110105108

Publisher's note Springer Nature remains neutral with regard to jurisdictional claims in published maps and institutional affiliations. 